\title{
Automated 3D Modeling of Buildings in Suburban Areas Based on Integration of Image and Height Data
}

\author{
Kourosh Khoshelham \\ Center of Excellence in Surveying Engineering and Disaster Management, \\ Dept. of Surveying and Geomatics Engineering, University of Tehran, \\ Iran.
}

\begin{abstract}
This paper presents an automated method for 3D modeling of buildings in suburban areas through the integration of image and height data. The method is based on matching a CAD model of the building against the image, while the selection of the CAD model relies on clues derived from height data. The matching procedure makes use of straight line segments extracted from the image and grouped on the basis of proximity and parallelism relations. For the selection of the model, a process of fitting planar faces to height data guided by a segmentation of image data is employed. Roof planes are recognized by taking into account the height of each plane over a DTM of the scene. The integration strategy proposed in this paper is capable of exploiting accurate and reliable information from both sources of data. Incomplete image regions can be refined by using clues from height data, and incomplete image edges can be efficiently handled in the model matching process. The reconstruction strategy takes advantage of the high accuracy of laser range data, but is not influenced by the low spatial resolution of the DSM. An experiment is conducted to evaluate the performance of the proposed approach. Results indicate the promising performance of the proposed approach in reconstructing buildings with an acceptable accuracy at a reasonable computational cost.
\end{abstract}

\section{Introduction}

Automated generation of 3D models of buildings in urban and suburban areas has long been an attractive research topic for scientists within the 
area of digital Photogrammetry and computer vision. A large number of methods have been developed to fully automate the process of building extraction. These methods mainly vary in the utilized source of input data and the type of output model. The input data that have been used for automated building extraction mostly include aerial imagery, laser range data, 2D maps and combinations of them. Recent developments show a trend towards integrating image and laser rang data. Complementary properties of image and laser range data suggest that these sources can be used as synergistic partners in automated building extraction. Aerial images can be obtained in a very high spatial resolution; however, 3D information extracted from such images is still very uncertain. In contrast, laser scanners provide accurate measurements of the terrain surface, but in a relatively low spatial resolution. This has led researchers to the idea of integrating image and height data for automated building extraction (Cord et al., 2001; Jaynes et al., 2003; Rottensteiner and Jansa, 2002; Rottensteiner et al., 2004).

In terms of output model, two major types of building models have been commonly used namely specific models and generic models. Specific models are parameterized CAD models, which are specific to the domain of buildings since they are tightly constrained with geometric constraints that most buildings represent. These constraints may limit the number of buildings that can be modeled; but in return, they guarantee that other objects cannot be modeled using this type of models. Modeling with parameterized models is often based on a hypothesize-and-verify strategy whereby a match is found between a number of hypothesized models and the features extracted from the data (Gruen, 1998; Jaynes et al., 2003; Khoshelham and Li, 2004). Generic models, on the other hand, are able to model a broader range of buildings because they represent looser constraints. The shortcoming of generic models is that other objects that satisfy the constraints might as well be modeled. Also, generic models carry no semantics about the type of the reconstructed building. Boundary representation with polyhedral models is the most commonly used method for generic modeling. In this method, planar faces are extracted from the image or height data and then stitched together to form a polyhedral model (Henricsson, 1998; Khoshelham, 2004; Vosselman, 1999). An intermediate type of model is the component-based model that consists of parameterized building parts with fixed geometry and unknown parameters. As component-based models cover a broader range of buildings, they have also been referred to as generic models (Braun et al., 1995; Heuel and Kolbe, 2001). Fig. 1. shows different types of models. 

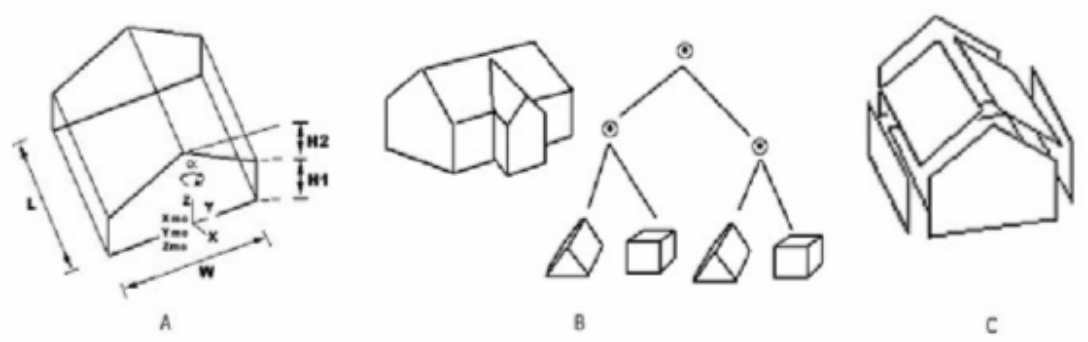

Fig. 1. Types of models. A. parameterized model; B. component-based model; C. polyhedral model.

The performance of the modeling with generic polyhedral models highly relies on the completeness of extracted features. This is because the correctness of extracted features cannot be verified due to the lack of tight constraints in generic models. Therefore, if extracted features are incorrect or some correct features are missed, the modeling process is likely to fail. Parameterized models enjoy a higher level of robustness because the presence of tight constraints and the known geometry of such models greatly reduce the sensitivity of the modeling process to incomplete features. This paper presents an automated method for modeling buildings in suburban areas with parameterized models based on the integration of image and laser range data. Parameterized models are suitable for suburban areas since buildings in suburbs often have simple shapes. The modeling process is based on matching one or more hypothesized models with straight line segments extracted from the image. The generation of hypotheses relies on a roof plane reconstruction process, which works on the basis of fitting planar faces to height data guided by a segmentation of image data.

The paper has six sections. Section 2 provides an overview of the proposed method. Section 3 describes the roof plane reconstruction process for the generation of model hypotheses. In section 4, the model matching process for the verification of the hypotheses and computation of the parameters of the correct model is discussed. Section 5 describes the experimental evaluation of the method. Conclusions are drawn in section 6 .

\section{An overview of the method}

In order to take advantage of parameterized models, a number of building models with fixed geometry are constructed and stored as sets of parameters in a library of models. The proposed method is basically a search for a 
correct match between one of the models in the library and the set of linear image features. This search can be performed in a brute-force fashion by exhausting the entire library and matching all the models against image features. In practice, however, an exhaustive search can be very expensive because model matching is a relatively costly process and the library may contain a very large number of building models. Therefore, we adopt a hypothesize-and-verify strategy to limit the search and speed up the matching process.

For the generation of model hypotheses, we employ a split-and-merge method that has been used for the reconstruction of building roofs from image and height data (Khoshelham, 2005; Khoshelham et al., 2005). In this method, first a segmentation of the image data is obtained. A plane fitting algorithm is then used to find planar surfaces in the height points within every image region. The plane fitting algorithm is followed by a split-and-merge process where incomplete image regions are refined and roof planes are identified. The number of the roof planes is used as an index to the library of parameterized models in order to select a limited number of model hypotheses.

The verification of the hypotheses is based on a model matching method (Lowe, 1987; Khoshelham and Li, 2004). In this method, the hypothesized models are matched against the set of straight line segments extracted from the image. In addition to model parameters, a fitness error is also computed for each match. A correct match is identified by examining the fitness error as well as computed parameters.

\section{Generation of model hypotheses}

For the generation of model hypotheses first a segmentation of the image is obtained. Every extracted image region is coupled with a number of height points from a last echo laser scanner DSM, which project to that region provided that both the image and DSM are referenced to a same datum. A robust regression method based on the least median of squares (LMS) (Rousseeuw and Leroy, 1987) is employed to fit planar surfaces to the height points contained in each image region. The LMS-type plane fitting algorithm is iteratively applied to each image region and its associated height points until no more planes are found. The split-and-merge process makes use of these detected planes to identify incomplete regions. If multiple planes are found in a single region, then the region is split according to the number of the planes. In case neighboring regions each have a single plane but their planes are coplanar, then the two regions are merged. In 
other words, the split-and-merge process establishes a one-to-one correspondence between image regions and planar surfaces in height data. Fig. 2 illustrates the process of plane fitting in an overgrown region.
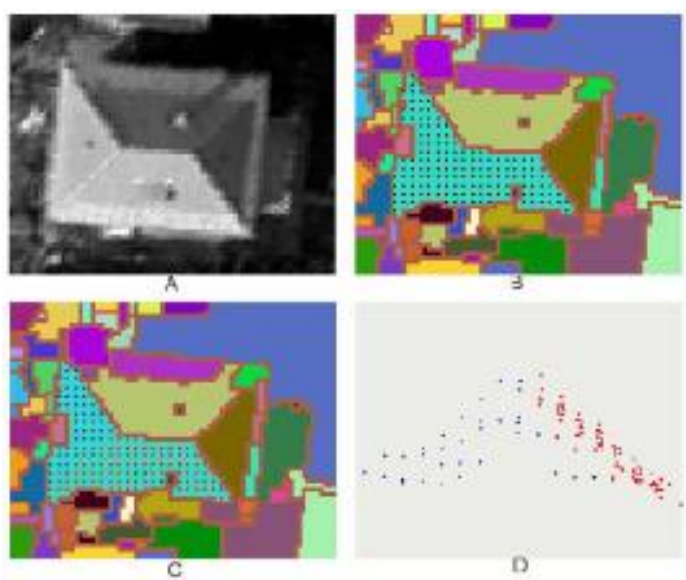

Fig. 2. Plane fitting process. A. A sample aerial image of a building; B. The initial segmentation of the image representing an overgrown region and its associated height points; C. Two planes are detected in the height points contained in the overgrown region (depicted with blue points and red points); D. The height points as seen from a different angle.

For every region-plane two average height values are derived, one from the DSM and one from a DTM of the scene. The difference between these two values indicates whether the region-plane belongs to a roof. Usually a threshold of about $2 \mathrm{~m}$ can be applied to separate roofs from non-roof planes. More details on the process of roof plane reconstruction can be found in Khoshelham (2005).

The number of the detected roof planes for each building serves to form the model hypotheses. As can be seen in the example model library of Fig. 3 , models in the library are categorized with respect to the number of roof planes. Therefore, having determined the number of roof planes, the search in the library will be narrowed down to only one class of building models. Usually all the models in the relevant class are hypothesized, although the slope of the roof planes can also be used to further reduce the number of hypotheses. 


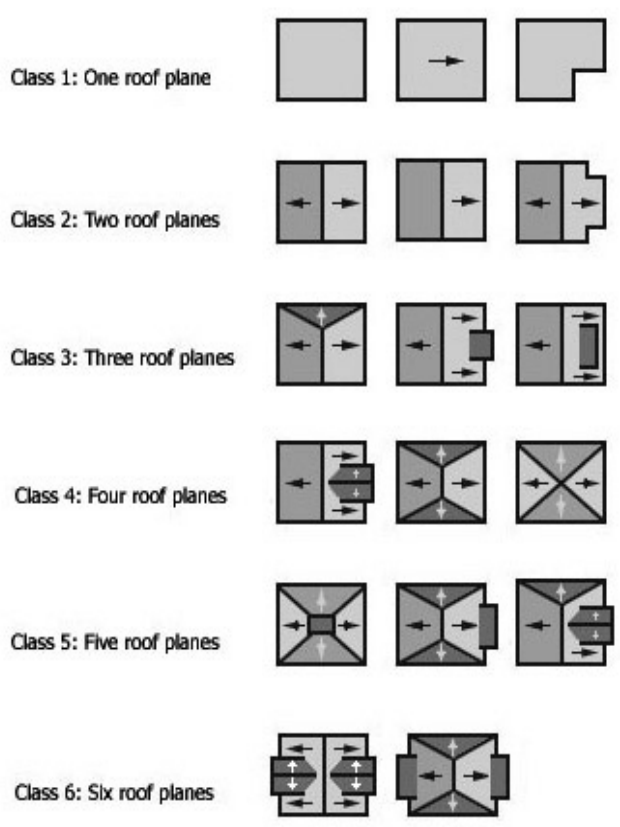

Fig. 3. An example of a model library in which models are categorized based on the number of roof planes (arrows denote slope).

\section{Verification of the hypotheses}

Model matching (Lowe, 1987) is an efficient method for the verification of the presence of a $3 \mathrm{D}$ object in a $2 \mathrm{D}$ image, provided that a model of the object is available. Having generated a number of model hypotheses, the model matching method can be used to verify the hypotheses and find the correct match. The matching algorithm requires corresponding lines in image and model to be selected beforehand. For the selection of image lines, a line extraction algorithm is applied to the image data. The extracted line segments undergo a perceptual grouping process, which selects a set of image lines that form a structure based on some perceptual relations such as proximity and parallelism. Fig. 4 demonstrates an example of the performance of perceptual grouping in the selection of a set of lines that are most relevant to a polyhedral object (here building) based on proximity and parallelism relations. 


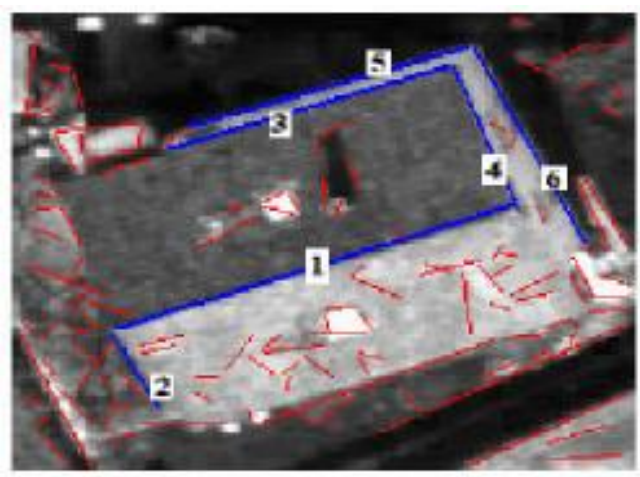

Fig. 4. Perceptual grouping of image lines based on proximity and parallelism relations.

The same perceptual relations are used to select the corresponding model lines. Every hypothesized model is rearranged as a set of lines in a graph structure interlinked with respect to proximity and parallelism relations. The selection of corresponding model lines is a search in this graph structure for those model lines that carry the same relations as the set of grouped image lines retains. Usually multiple sets of model lines satisfy the proximity and parallelism criteria, resulting in an increase in the number of hypotheses.

Some properties of the grouped image lines, e.g. the length and the orientation of the longest line in the group, can be exploited for the computation of initial values for the parameters of the hypothesized models. Once the corresponding image and model lines are selected and the initial values for the model parameters are computed, the set of model lines is projected to the image space and the distance between the endpoint of every model line to the corresponding image line is computed. With the computed distances a set of observation equations is formed that contain the parameters of the model as unknowns. These unknown model parameters are estimated in a least squares adjustment that minimizes the sum of squared distances.

The estimated parameters for every hypothesized model and its selected sets of model lines, in addition to a fitness error computed as the mean of distance residuals after the adjustment, form the basis for the verification of the hypotheses. When an incorrect hypothesis is matched, the least squares adjustment will usually result in implausible estimations for the unknown parameters (e.g. negative length or width). The fitness error will also be high in the case of an incorrect match. A successful match is found 
as one with plausible parameters and a minimum fitness error. Further details on the application of model matching to the verification of hypotheses can be found in Khoshelham and $\mathrm{Li}$ (2004).

\section{Experimental results}

A set of data consisting of an aerial orthoimage, a laser scanner DSM and a DTM of a suburban part of Memingen city, Germany, was obtained for the experimental evaluation of the method. The orthoimage was resampled by the supplier with a pixel size of $0.5 \mathrm{~m}$, while the height data had a $1.0 \mathrm{~m}$ ground resolution. Fig. 5 shows the orthoimage and the last echo DSM of the scene. A segmentation of the image was obtained using a morphological watershed algorithm. The split-and-merge process was then applied to the extracted image regions and roof region-planes were reconstructed. Fig. 6 depicts the reconstructed roof region-planes. As can be seen, in most cases roof planes are correctly reconstructed, although there are cases where roof planes are missed or some region-planes are wrongly detected as roof planes. The results of the roof reconstruction process are summarized in Table 1. The numbering of the buildings is shown in Fig.7.

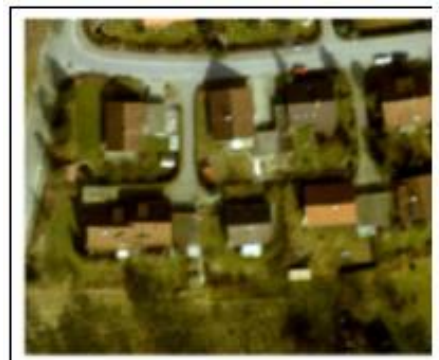

A

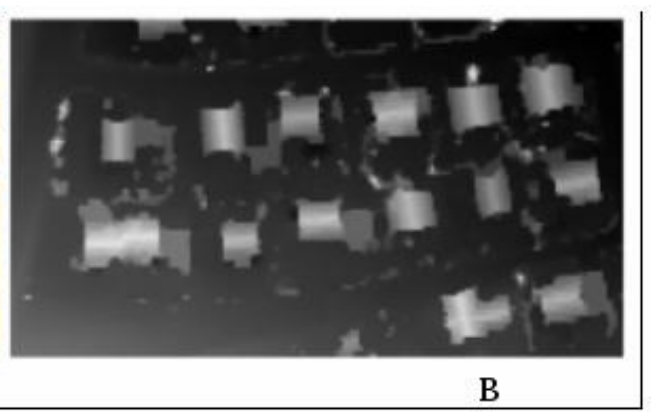

Fig. 5. A. Aerial orhtoimage of the scene of experiment; B. Last echo laser scanner DSM of the scene. 


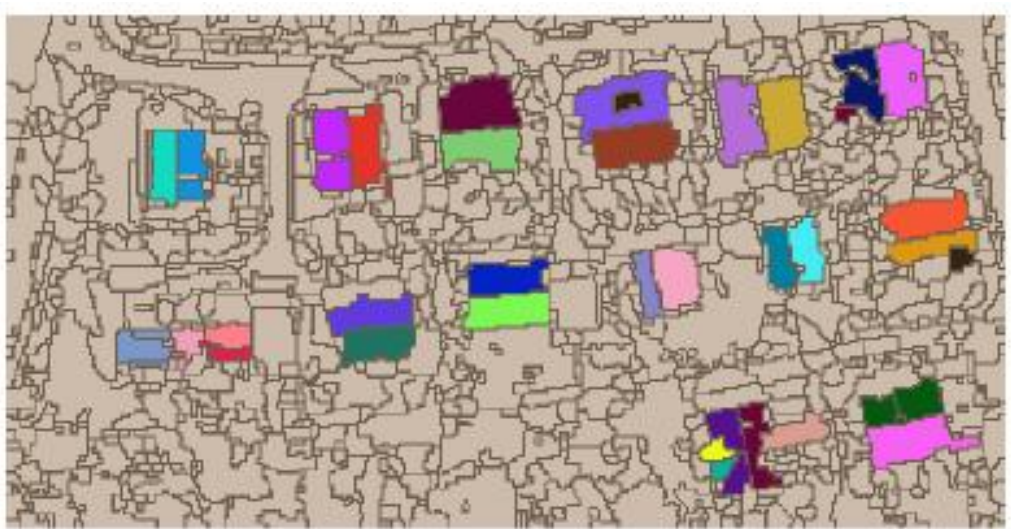

Fig. 6. Roof region-planes reconstructed in the split-and-merge process

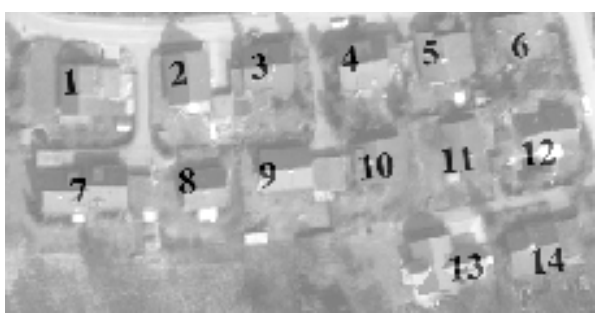

Fig. 7. Numbering of buildings

Table 1. Peformance of roof plane reconstruction process

\begin{tabular}{lllllllllllllll}
\hline Bldg. No. & 1 & 2 & 3 & 4 & 5 & 6 & 7 & 8 & 9 & 10 & 11 & 12 & 13 & 14 \\
\hline $\begin{array}{l}\text { Total No. of } \\
\text { roof planes }\end{array}$ & 2 & 2 & 2 & 3 & 2 & 2 & 6 & 2 & 2 & 2 & 2 & 2 & 6 & 2 \\
\hline $\begin{array}{l}\text { No. of detected } \\
\text { planes }\end{array}$ & 2 & 2 & 2 & 3 & 2 & 3 & 4 & 2 & 2 & 2 & 2 & 3 & 5 & 2 \\
\hline $\begin{array}{l}\text { No. of missed } \\
\text { roof planes }\end{array}$ & 0 & 0 & 0 & 0 & 0 & 0 & 3 & 0 & 0 & 0 & 0 & 0 & 1 & 0 \\
\hline $\begin{array}{l}\text { No. of planes } \\
\text { wrongly de- } \\
\text { tected as roof }\end{array}$ & 0 & 0 & 0 & 0 & 0 & 1 & 3 & 0 & 0 & 0 & 0 & 1 & 0 & 0 \\
\hline
\end{tabular}

The reconstructed roof planes served for the generation of hypotheses. All hypothesized models were matched against the grouped image lines and verified according to the result of the matching. Model matching was 
carried out in 2D since the 3D parametric forms of the roof planes were already derived and only $2 \mathrm{D}$ roof boundaries were unknown. Table 2 summarizes the fitness errors computed for the successful matches. As expected, a correct match was not found in cases where roof planes were not correctly reconstructed. Fig. 8 shows the roof models found in the matching process. It can be observed that the performance of the model matching algorithm is not influenced by the incompleteness of the image lines because perpendicular distances are used as observations in the least squares estimation model. A visualization of the reconstructed 3D models is shown in Fig. 9.

Table 2. Fitness errors for successful matches

\begin{tabular}{|c|c|c|c|c|c|c|c|c|c|c|c|c|c|}
\hline Bldg. No. & 12 & 23 & 4 & 5 & 6 & 7 & 8 & 9 & 10 & 11 & 12 & 13 & 14 \\
\hline $\begin{array}{l}\text { Matching fitness er- } \\
\text { ror (pixels) }\end{array}$ & $\stackrel{0}{\dot{\omega}} \dot{8}$ & 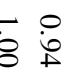 & $\stackrel{0}{\infty}$ & i̊ & & & $\dot{8}$ & \% & $\dot{\varnothing}$ & $\dot{8}$ & ' & & i̊ \\
\hline
\end{tabular}



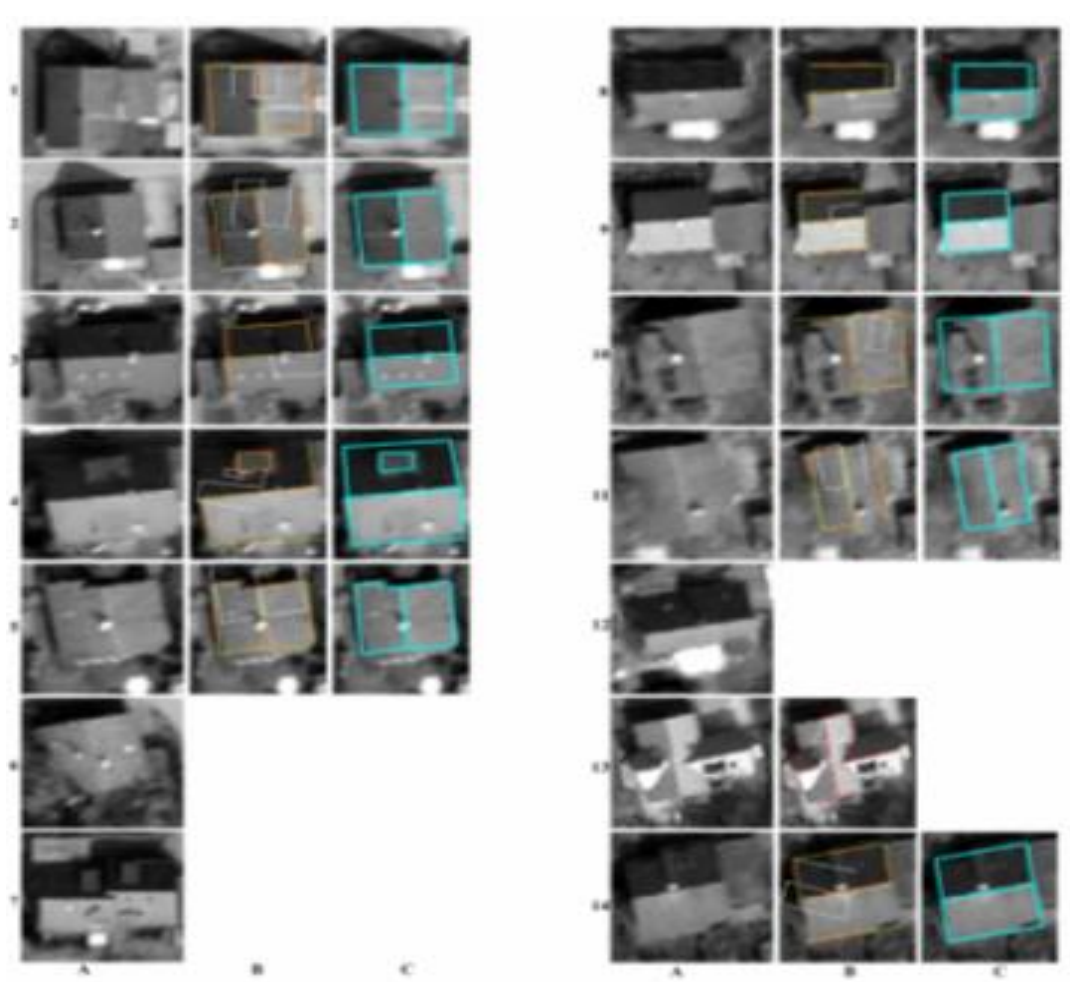

Fig. 8. Roof matching process. A. Original images; B. Grouped image lines (in red), corresponding lines of the initialized roof model (in white), and matched model lines in the last iteration (in yellow) for the successful matches; C. Complete roof models overlaid on images. 


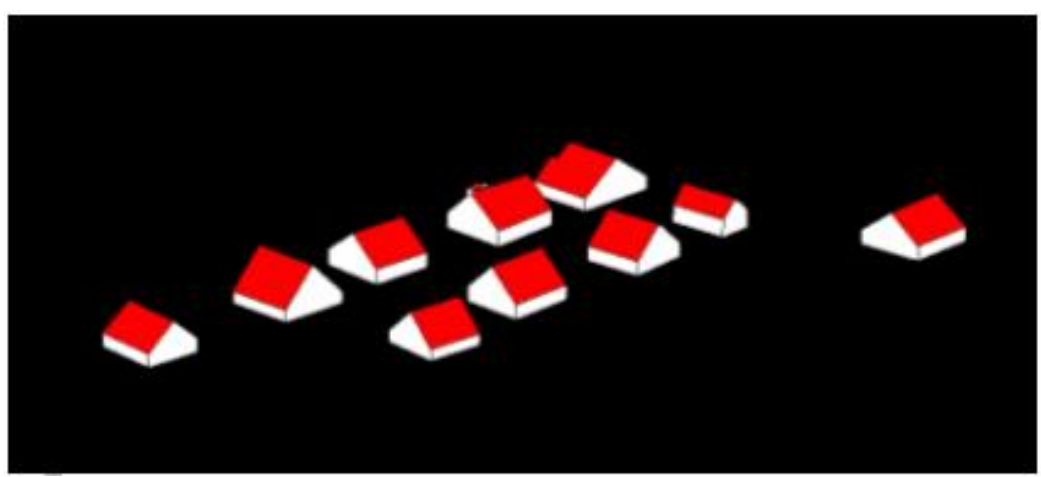

Fig. 9. 3D visualization of the reconstructed models.

\section{Conclusions}

In this paper a method was presented for automated $3 \mathrm{D}$ reconstruction and modeling of buildings from aerial image and laser range data. The method was shown to be capable of exploiting accurate and reliable information from both sources of data. Incomplete image regions can be refined by using clues from height data, and incomplete image edges can be efficiently handled in the model matching process. The reconstruction strategy takes advantage of the high accuracy of laser range data, but is not influenced by the low spatial resolution of the DSM. The experimental results also indicate the promising performance of the proposed strategy in automated 3D reconstruction of buildings in a suburban area.

\section{References}

Braun, C. et al., 1995. Models for photogrammetric building reconstruction. Computers \& Graphics, 19(1): 109-118.

Cord, M., Jordan, M. and Cocquerez, J.-P., 2001. Accurate building structure recovery from high resolution aerial imagery. Computer Vision and Image Understanding, 82: 138-173.

Gruen, A., 1998. TOBAGO -- a semi-automated approach for the generation of 3D building models. ISPRS Journal of Photogrammetry and Remote Sensing, 53(2): 108-118.

Henricsson, O., 1998. The Role of Color Attributes and Similarity Grouping in 3D Building Reconstruction. Computer Vision and Image Understanding, 72(2): 163-184. 
Heuel, S. and Kolbe, T.H., 2001. Building reconstruction: the dilemma of generic versus specific models. Künstliche Intelligenz, 3: 57-62.

Jaynes, C., Riseman, E. and Hanson, A., 2003. Recognition and reconstruction of buildings from multiple aerial images. Computer Vision and Image Understanding, 90(1): 68-98.

Khoshelham, K., 2004. Building extraction from multiple data sources: a data fusion framework for reconstruction of generic models. International Archives of Photogrammetry and Remote Sensing, 35(B3): 980-986.

Khoshelham, K., 2005. Region refinement and parametric reconstruction of building roofs by integration of image and height data. In: U. Stilla, F. Rottensteiner and S. Hinz (Editors), CMRT05, Vienna, Austria, pp. 3-8.

Khoshelham, K. and Li, Z.L., 2004. A model-based approach to semi-automated reconstruction of buildings from aerial images. Photogrammetric Record, 19(108): 342-359.

Khoshelham, K., Li, Z.L. and King, B., 2005. A split-and-merge technique for automated reconstruction of roof planes. Photogrammetric Engineering and Remote Sensing, 71(7): 855-862.

Lowe, D.G., 1987. Three-dimensional object recognition from single twodimensional images. Artificial intelligence, 31(3): 355-395.

Rottensteiner, F. and Jansa, J., 2002. Automatic extraction of buildings from LIDAR data and aerial images, International Archives of Photogrammetry and Remote Sensing, Volume XXXIV / 4, pp. 569-574.

Rottensteiner, F., Trinder, J., Clode, S. and Kubik, K., 2004. Fusing airborne laser scanner data and aerial imagery for the automatic extraction of buildings in densely built-up areas. International Archives of Photogrammetry and Remote Sensing, 35(B3): 512-517.

Rousseeuw, P.J. and Leroy, A.M., 1987. Robust regression and outlier detection. John Wiley \& Sons, New York, 329 pp.

Vosselman, G., 1999. Building reconstruction using planar faces in very high density height data, ISPRS Conference on Automatic Extraction of GIS Objects from Digital Imagery, Munich, pp. 87-92. 
